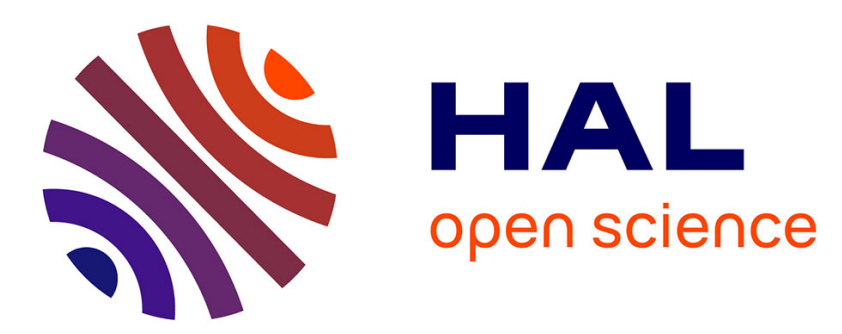

\title{
Expression of long non-coding RNA ANRIL predicts a poor prognosis in intrahepatic cholangiocarcinoma
}

Gaëlle Angenard, Aude Merdrignac, Corentin Louis, Julien Edeline, Cédric

Coulouarn

\section{- To cite this version:}

Gaëlle Angenard, Aude Merdrignac, Corentin Louis, Julien Edeline, Cédric Coulouarn. Expression of long non-coding RNA ANRIL predicts a poor prognosis in intrahepatic cholangiocarcinoma. Digestive and Liver Disease, 2019, 51 (9), pp.1337-1343. 10.1016/j.dld.2019.03.019 hal-02152868

HAL Id: hal-02152868

https://hal-univ-rennes1.archives-ouvertes.fr/hal-02152868

Submitted on 15 Jul 2019

HAL is a multi-disciplinary open access archive for the deposit and dissemination of scientific research documents, whether they are published or not. The documents may come from teaching and research institutions in France or abroad, or from public or private research centers.
L'archive ouverte pluridisciplinaire HAL, est destinée au dépôt et à la diffusion de documents scientifiques de niveau recherche, publiés ou non, émanant des établissements d'enseignement et de recherche français ou étrangers, des laboratoires publics ou privés. 
Expression of long non-coding RNA ANRIL predicts a poor prognosis in intrahepatic cholangiocarcinoma

Gaëlle Angenard ${ }^{\mathrm{a}}$, Aude Merdrignac, $\underline{\mathrm{MD}}^{\mathrm{a}}$, Corentin Louis, $\underline{\mathrm{MSc}}^{\mathrm{a}}$, Julien Edeline, $\underline{\mathrm{MD}, \mathrm{PhD}}{ }^{\mathrm{a}}$ and Cédric Coulouarn, $\underline{\mathrm{PhD}}^{\mathrm{a}}$

a Inserm, Univ Rennes, Inra, Institut NuMeCan (Nutrition Metabolisms and Cancer), UMR_S 1241, CHU Rennes, Centre de Lutte contre le Cancer Eugène Marquis, Rennes, France

Correspondance to: Dr. Cédric Coulouarn, Inserm UMR_S 1241, CHU Pontchaillou, 2 rue Henri Le Guilloux, F-35033 Rennes, France.

Phone +332232338 81; Fax +33299540137

E-mail: cedric.coulouarn@inserm.fr

Running title: ANRIL expression in cholangiocarcinoma

Electronic Word Count: $\underline{2826}$ words

Funding: This work was supported by Inserm, Université de Rennes 1, INCa, and ITMO Cancer AVIESAN (Alliance Nationale pour les Sciences de la Vie et de la Santé) dans le cadre du Plan cancer (Non-coding RNA in cancerology: fundamental to translational; [Grant C18007NS to CC]). CL is supported by a PhD fellowship from Université de Rennes 1. 


\section{Abstract}

Background: Intrahepatic cholangiocarcinoma (iCCA) is a deadly cancer worldwide associated with an increased incidence, limited therapeutic options and absence of reliable prognostic biomarkers. Long non-coding RNAs (IncRNA) emerge as relevant biomarkers in cancer being associated with tumor progression. However, IncRNA have been poorly investigated in iCCA.

Aim: To identify IncRNA significantly associated with the survival of patients with iCCA after tumor resection for curative intent.

Methods: Gene expression profiling and Q-RT-PCR were performed from a cohort of 39 clinically well-annotated iCCA. Univariate Cox proportional hazards model with Wald Statistic was used to identify IncRNA significantly associated with overall (OS) and/or disease-free (DFS) survival.

Results: A signature made of 9 IncRNA was identified to be significantly $(P<0.05)$ associated with OS and DFS, including 4 IncRNA (Inc-CDK9-1, XLOC_I2_009441, CDKN2B-AS1, HOXC13AS) highly expressed in poor prognosis iCCA and 5 IncRNA (Inc-CCHCR1-1, Inc-AF131215.3.1, Inc-CBLB-5, COL18A1-AS2, Inc-RELL2-1) highly expressed in better prognosis iCCA. We further validated CDKN2B-AS1 (ANRIL) as a poor prognosis biomarker, not only in iCCA, but also in hepatocellular carcinoma, kidney renal clear cell carcinoma and uterine corpus endometrial carcinoma.

Conclusions: We report a prognosis IncRNA signature in iCCA and the clinical relevance of CDKN2B-AS1 (ANRIL) overexpression in several cancers.

\section{Keywords}

cholangiocarcinoma; prognosis; long non-coding RNA; ANRIL 


\section{Introduction}

Cholangiocarcinomas (CCA) comprise several types of tumors arising from the malignant transformation of cholangiocytes lining different part of the biliary tree. Based on the anatomical location, intrahepatic (iCCA), perihilar ( $\mathrm{pCCA}$ ) and distal (dCCA) CCA are recognized ${ }^{1,2}$. Each of these CCA subtypes exhibits specific biological and pathological features, e.g. in term of clinical presentation, risk factors, prognosis and therapeutic strategies ${ }^{2}$. In the liver, iCCA represents the second most common type of malignant primary tumor after hepatocellular carcinoma (HCC), encompassing $10 \%$ to $15 \%$ of cases. However, an increase in iCCA incidence and mortality has been observed worldwide over the last decades. Today, surgical resection still remains the best curative treatment strategy of CCA, although it is associated with a high risk of tumor recurrence ${ }^{1}$. For patients with unresectable or metastatic CCA, mostly as a result of a late diagnosis, systemic chemotherapy (usually a gemcitabine and cisplatin combination) is proposed as a palliative treatment ${ }^{1}$. Recently, adjuvant chemotherapy was demonstrated effective in resected $\mathrm{CCA}{ }^{3}$. However, defining the optimal population that could benefit from adjuvant chemotherapy is particularly important. From a clinical point of view, the identification of innovative biomarkers for early diagnosis, prognosis and response to therapies, may improve the management and the survival of patients with iCCA. Although carbohydrate antigen 19-9 (CA19-9) and carcinoembryonic antigen (CEA) levels may be elevated in some patients, there is no specific serum biomarkers for the diagnosis of iCCA. Several prognostic tissue biomarkers have been proposed based on genomic analyses. Notably, by combining laser capture microdissection and unsupervised gene expression profiling, we previously established clinically relevant signatures specific of the tumor microenvironment in iCCA ${ }^{4}$. Thus, an increased expression of osteopontin (OPN) and lysyl oxidase like 2 (LOXL2) in the stroma was identified as a significant prognostic factor associated with a reduced survival of patients after tumor resection ${ }^{4,5}$. More recently, we identified a novel long non-coding RNA (IncRNA) up-regulated 
by the transforming growth factor beta (TGF $\beta$ ) in human iCCA and associated with an inflammatory microenvironment, indicating that IncRNAs are also actively involved in iCCA carcinogenesis ${ }^{6}$.

LncRNAs belong to an emerging class of regulatory RNAs that play critical roles in modulating a large variety of biological processes and cell signaling pathways. Accordingly, altered IncRNA expression has been reported in several cancers, including $\mathrm{CCA}^{7}$. Recent meta-analyses of The Cancer Genome Atlas (TCGA) database identified differentially expressed IncRNA in CCA (e.g. LINC00313, NEXN-AS1, COL18A1-AS1, HULC), the expression of some of them being significantly associated with overall survival (OS) of patients ${ }^{8,9}$. Mechanistically, co-expression network analysis of coding and non-coding RNA in resected CCA tumors identified IncRNA (e.g. APOC1P1, PVT1) possibly regulating inflammation and oxidative stress in malignant cholangiocytes ${ }^{10,11}$. Notably, pvt1 oncogene (PVT1) was shown to promote cell proliferation and migration by epigenetically silencing angiopoietin like 4 (ANGPTL4), a tumor suppressor gene candidate $^{12}$. Cyclin dependent kinase inhibitor 1A (CDKN1A), another well-described tumor suppressor gene, was shown to be epigenetically silenced in CCA through the interaction of small nucleolar RNA host gene 1 (SNHG1) IncRNA with enhancer of zeste 2 (EZH2), the catalytic subunit of the polycomb repressive complex 2 (PRC2) ${ }^{13}$. Nuclear paraspeckle assembly transcript 1 (NEAT1) IncRNA may interact with EZH2 as well to repress the expression of E-cadherin ( $\mathrm{CDH1}$ ), thus promoting epithelial-to-mesenchymal transition (EMT) and metastasis ${ }^{14}$. Accordingly, an increased expression of SNHG1 and NEAT1 has been associated with aggressive tumorigenesis features in CCA ${ }^{13,14}$. NEAT1 has been also reported to contribute to gemcitabine sensitivity ${ }^{15}$. Conversely, IncRNA may positively affect the expression of oncogenic regulators, either directly or indirectly. Thus, epigenetically-induced IncRNA-1

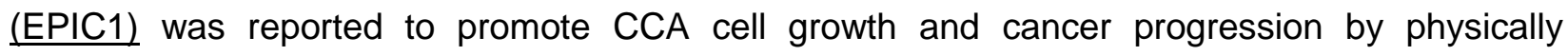
interacting with MYC oncogene and possibly acting as a guide to direct MYC onto its target 
genes involved in cell cycle progression ${ }^{16}$. Interestingly, LINC01296 IncRNA was demonstrated to stabilize MYCN oncogene by sponging microRNA-5095, which naturally targets MYCN mRNA for degradation ${ }^{17}$. Urothelial cancer associated 1 (UCA1) IncRNA was reported to promote CCA formation and progression by activating a proliferative AKT/GSK-3 $\beta / C C N D 1$ axis and by inducing EMT, migration and invasion ${ }^{18}$. Metastasis associated lung adenocarcinoma transcript 1 (MALAT1), a IncRNA induced in several cancers, promotes cell proliferation and invasion of CCA cells by activating the PI3K/AKT pathway as well ${ }^{19}$. While interesting mechanisms of IncRNA action have been elegantly described in CCA carcinogenesis, reports on their clinical relevance are scarce. The expression of few individual IncRNA (e.g. CRNDE, UCA1, TUG1) was reported to correlate with clinical progression (e.g. tumor grade, lymph node metastasis, TNM stage) and was identified as risk factors for an unfavorable overall survival (OS) in iCCA ${ }^{16,18,20-23}$. Circulating IncRNAs, including prostate cancer associated transcript 1 (PCAT1) and MALAT1, have been also reported in the plasma of patients with $\mathrm{pCCA}^{24}$. However, besides individual IncRNA, there is no exhaustive report on the clinical relevance of IncRNA expression as prognostic factors in iCCA so far ${ }^{7,23}$. Thus, this study was specifically designed to establish an unsupervised robust signature of IncRNA predictive of overall (OS) and/or disease-free (DFS) survival, by using pan-genomic microarrays and a cohort of clinically well-annotated iCCA.

\section{Methods}

\section{Human iCCA samples}

A cohort of 39 patients with iCCA was studied. Freshly frozen tumor samples were obtained through the French liver cancer biobanks network - INCa (BB-0033-00085). These patients underwent liver tumor resection for curative intent from January 2007 to July 2014 . None of the patient underwent liver transplantation. Six patients underwent chemo- and/or radio-therapy prior surgery. For all cases, iCCA diagnosis was confirmed by a pathological examination. Tissue sections were evaluated after hematoxylin-eosin and Sirius Red staining (SI Figure 1). Written 
informed consent was obtained from each patient included in the study. The study protocol conforms to the ethical guidelines of the 1975 Declaration of Helsinki (6th revision, 2008), as reflected in a priori approval by the local institution's human research committee and the institutional review board of Inserm (IRB00003888, IORG0003254). Clinical and pathological features of patients are reported in Table 1.

\section{Gene expression profiling}

Total RNA was extracted from tumor tissue sections and purified with a miRNAeasy kit (Qiagen, Courtaboeuf, France). Genome-wide expression profiling was performed using the low-input QuickAmp labeling kit and human SurePrint G3 8x60K pangenomic microarrays (Agilent Technologies, Santa Clara, USA). Gene expression data were processed using Feature Extraction and GeneSpring softwares (Agilent Technologies), as previously described ${ }^{6}$. Clustering analysis was performed using Gene Cluster and TreeView softwares ${ }^{25}$.

\section{$Q-R T-P C R$}

Quantitative-RT-PCR was performed by using a SYBR Green master mix (Applied Biosystems, Carlsbad, CA) as previously described ${ }^{6}$. A mixture of oligo-dT (250ng) and random hexamers (100ng) was used to prime the reverse transcription of $1 \mu \mathrm{g}$ total RNA (Superscript III RT, Invitrogen, Carlsbad, CA). Quantitative analysis of PCR data was conducted with the $2^{-\Delta \Delta C t}$ method using actin beta (ACTB) Ct values for normalization. Melting analysis was conducted to validate the specificity of PCR products. PCR analysis was performed using the following primers: CDKN2B-AS1-Forward, 5'-TCCAGTGCAAGTATGGTCTGT-3'; CDKN2B-AS1-Reverse, 5'-TAGCAGAAAGCTGCAAAGGC-3'; ACTB-Forward, 5'-GGACTTCGAGCAAGAGATGG-3';

\section{ACTB-Reverse, 5'-AGCACTGTGTTGGCGTACAG-3'.}

\section{Statistical analysis}

Statistical analysis of microarray data was as previously described ${ }^{26}$. Notably, IncRNAs significantly associated with OS and/or DFS were identified using an univariate Cox proportional 
hazards model with Wald Statistic ${ }^{26,27}$. Up- and down-regulated IncRNA were determined by the ration of their expression in poor versus better prognosis iCCA. A meta-analysis of RNAsequencing data generated by The Cancer Genome Atlas (TCGA) was also performed to determine the clinical relevance of CDKN2B-AS1/ANRIL expression in independent datasets. The Kaplan-Meier method was used to estimate OS and DFS, and group differences were analyzed with the log-rank test ${ }^{4,5,28}$.

\section{Results}

\section{Patient characteristics}

Thirty nine patients (27 men) who underwent liver resection with curative intent for iCCA were included in the profiling study (Table 1 ). The median age was $61 \pm 14$ years. Similar to our previous cohort ${ }^{5}, 69 \%$ patients had a tumor with a size $>5 \mathrm{~cm}$. The cohort included $15(38 \%)$ well-differentiated, 17 (44\%) moderately-differentiated, and 7 (18\%) poorly-differentiated tumors. Satellite nodules were detected in $49 \%$ patients and lymph nodes invasion in $28 \%$ patients.

\section{A IncRNA signature predictive of OS and DFS}

Gene expression profiling was performed on RNA extracted from the 39 iCCA cases. In order to identify innovative prognostic biomarkers, statistical analysis was restricted to the differential expression of IncRNAs. By using an univariate Cox proportional hazards model with Wald Statistic, 16 and 17 IncRNAs were identified to be significantly $(P<0.05)$ associated with OS and DFS, respectively (Figure $1 \mathrm{~A}$ and Table 2). Among those, 9 were predictive of both OS and DFS, including 4 up- and 5 down-regulated IncRNA in poor prognosis iCCA (Figure $1 \mathrm{~A}$ and Table 2). CDKN2B-AS1 and COL18A1-AS2 are two examples of such prognostic IncRNAs. Indeed, CDKN2B-AS1 median expression was able to segregate iCCA tumors into two groups, where a high expression of CDKN2B-AS1 was significantly associated with a reduced OS $(P=0.008)$ and DFS $(P=0.002)$. Conversely, highly COL18A1-AS2 expressing iCCA tumors were significantly associated with a better prognosis (Figure 1B). In addition, clustering analysis of 39 
iCCA tumors based on the expression of the 9 IncRNA expression signature identified two clusters: cluster A and cluster B (Figure 1C). Validating this prognostic IncRNA signature, patients included in clusters A and B exhibited a significant difference in OS and DFS, as demonstrated by Kaplan Meier plot analysis and log-rank testing (Figure 1D). Cluster A was also associated with a significantly higher number of poorly-differentiated iCCA (SI Table 1).

\section{CDKN2B-AS1 is a poor prognosis biomarker in cancer}

Quantitative RT-PCR was performed to validate the microarray data. We focused on CDKN2BAS1 that exhibited a high variability among the samples and the more robust statistical significance (Figure $1 \mathrm{C}$ and Table 2). Thus, from our initial cohort of $39 \mathrm{iCCA}$, two groups were defined based on the expression of CDKN2B-AS1, including 15 highly expressing tumors and 15 tumors with low or no CDKN2B-AS1 expression (Figure 2A). Validating our previous observation, a marked difference was observed in the OS of these two groups of patients (Figure 2A). No significant association with other clinical and pathological variables was identified in our cohort of iCCA (SI Table 2). More interestingly, a high expression of CDKN2BAS1 was shown to be significantly $(P<0.05)$ associated with a reduced survival of patients with hepatocellular carcinoma, kidney renal clear cell carcinoma and uterine corpus endometrial carcinoma (Figure $2 \mathrm{~B}$ ), identifying CDKN2B-AS1 as a poor prognosis biomarkers in several cancers.

\section{Discussion}

CCA is a deadly cancer worldwide waiting for innovative biomarkers and therapeutic strategies to improve the survival of patients ${ }^{1,2}$. In iCCA, several clinical variables have been identified as important prognostic factors after surgical resection for curative intent, including tumor size, pathological lymph node involvement and vascular invasion 1,29,30. Omics approaches (e.g. genomics, proteomics, metabolomics) have been extensively used to screen for novel candidate serum, bile and tissue molecular biomarkers in iCCA ${ }^{30}$. As an example, by proteomic 
analysis, a high expression of SSP411 in the serum and/or the bile has been significantly associated with iCCA diagnosis ${ }^{31}$. Serum and bile CA 19-9 level has been also associated with lymph node metastasis and OS although the specificity and sensitivity was highly heterogeneous ${ }^{30}$. By focusing more specifically on the alterations of the gene expression in the stroma of iCCA 4,32, we previously identified and validated specific mRNA and associated proteins, including OPN, LOXL2 and epithelial cell adhesion molecule (EPCAM), as independent prognostic tissue biomarkers associated with a reduced OS of patients with iCCA after tumor resection ${ }^{4,5,28}$. Here, we specifically focused on the expression of IncRNA and we identified a set of IncRNA predictive of OS $(n=16)$ and/or DFS ( $n=17)$, among those 9 were predictive of both OS and DFS. Except for HAND2-AS1, HOXC13-AS, and CDKN2B-AS1, the identified IncRNA were poorly characterized. Our data indicated that HAND2-AS1 down-regulation in poor prognosis iCCA is predictive of DFS. Accordingly, HAND2-AS1 has been recently shown to inhibit cancer cell proliferation, migration, and invasion in esophagus squamous cell carcinoma and colorectal cancer by sponging oncogenic microRNA, including miR-21 ${ }^{33,34}$. HOXC13-AS (up-regulated in poor prognosis iCCA from our IncRNA signature) has been shown to positively affects cell proliferation and invasion in nasopharyngeal carcinoma by sponging miR-383-3p ${ }^{35}$. CDKN2BAS1, also known as antisense non-coding RNA in the INK4 locus (ANRIL), was identified as a prognostic biomarker, not only in iCCA, but also in hepatocellular, kidney and uterine corpus carcinomas. Very promisingly, the low expressing group in iCCA showed a very high long term survival. In clinical context, one can question the need for adjuvant chemotherapy in a population with such high survival, potentially sparing toxicity for patients who do not require such therapy.

ANRIL is located at the CDKN2A/B genomic locus on chromosome 9p21.3 in human ${ }^{36}$. Interestingly, this locus is associated with an increased risk of cancer and metabolic disease, including type 2 diabetes, obesity and cardiovascular diseases ${ }^{37}$. ANRIL expression and function have been extensively investigated in several cancers but not in iCCA ${ }^{38}$. Here, we 
demonstrated that ANRIL expression is associated with a dismal outcome. Although clinically relevant differences in the expression of ANRIL expression have been highlighted in iCCA tumor tissues in our study, it remains to be determined whether ANRIL is up-regulated in iCCA as compared to normal biliary epithelium, as it was previously reported in other cancers, including $\underline{\text { HCC }^{39} \text {. Such an induction of ANRIL may suggest a key role in iCCA carcinogenesis. Indeed, }}$ gain and loss of function experiments in cancer cells demonstrated that ANRIL IncRNA favors cell proliferation and invasion. In vivo, ANRIL expression is associated with an increase of tumor size and growth, as well as invasive and metastatic phenotypes. Mechanistically, ANRIL was initially shown to promote cell proliferation and to inhibit apoptosis by epigenetically silencing the CDKN2A/B genomic locus ${ }^{40}$. In non-small cell lung cancer ANRIL was reported to silence kruppel like factor 2 (KLF2) and CDKN1A/P21 expression as well ${ }^{41}$. In gastric cancer, the impact of ANRIL on proliferation and apoptosis was mediated by the epigenetic silencing of miR99a and miR-449a, two miRNAs known to target mTOR and CDK6/E2F1 pathways ${ }^{42}$. In ovarian ${ }^{43}$ and bladder ${ }^{44}$ cancers, ANRIL may induce BCL2 apoptosis regulator (BCL2) and repress CDKN2B/P15, BCL2 associated $\mathrm{X}$, apoptosis regulator (BAX) and cleaved caspase-9, to promote cell cycle progression and inhibit apoptosis and senescence. In nasopharyngeal carcinoma, pro-tumorigenic features of ANRIL were associated with a glucose metabolism reprograming and an induction of side-population stem-like cancer cells ${ }^{45}$. In thyroid cancer, ANRIL promotes tumor cell invasion and metastasis through TGF- $\beta /$ Smad signaling and EMT ${ }^{46}$. In HCC, sponging of liver enriched miR-122-5p by ANRIL induces cell proliferation, metastasis and invasion ${ }^{47}$. Interestingly, ANRIL was reported to contribute to paclitaxel resistance in lung cancer, partly through poly(ADP-ribose) polymerase (PARP) and BCL2 modulating mitochondrial pathway ${ }^{48}$.

The clinical relevance of ANRIL expression has been evaluated in several types of cancers. Thus, a high ANRIL expression has been associated with TNM stage, advanced lymph node metastasis, tumor size, and was highlighted as an independent predictor of OS and/or DFS, 
particularly in nasopharyngeal ${ }^{45}$, ovarian ${ }^{43}$ or lung ${ }^{41,49}$ cancers. Interestingly, it was recently reported that ANRIL was significantly up-regulated in lung cancer tissues and serum samples compared with normal controls, suggesting that ANRIL may serve as a relevant circulating IncRNA biomarker ${ }^{50}$. Given the significant association of ANRIL and prognosis factors, it would be interesting to evaluate its expression in body fluids, including serum, urine and/or bile. Another point of interest is to understand the mechanisms by which the expression of ANRIL is increased in poor prognosis tumors. External risk factors, including lifestyle, nutrition or environment, have been shown to influence the expression of IncRNA ${ }^{51}$. Thus, HOTAIR and MALAT1 have been the most studied IncRNA in the context of tobacco use in lung cancer ${ }^{51}$. However, in our analysis, no statistically significant association was identified between the expression of ANRIL and lifestyle-associated factors (e.g. obesity, diabetes, tobacco, alcohol). Interestingly, ANRIL was shown to be transcriptionally induced by the cell cycle associated E2F1 transcription factor ${ }^{42}$, notably in an ATM-dependent manner following DNA damage ${ }^{52}$, suggesting a positive regulation in highly proliferative tumors. In addition, MYC oncogene was shown to positively regulate ANRIL expression in lung cancer ${ }^{53}$. Epigenetic regulation of ANRIL through promoter methylation has been also reported ${ }^{54}$.

Altogether, these data suggest that ANRIL may represent not only a clinically relevant biomarker for cancer diagnosis, prognosis and response to treatment, but also an innovative therapeutic target due to the well described pro-tumorigenic features of this IncRNA.

\section{Acknowledgements}

The authors thank the core facilities of Université de Rennes 1, Biosit (US18, UMS 3480) and Biogenouest (CRB Santé BB-0033-00056, GEH and H2P2), and the French liver cancer biobanks network - Institut National du Cancer (INCa) BB-0033-00085, including Beaujon, Bordeaux, Mondor, Nantes, Paris-Sud and Rennes biobanks. CC and JE are members of the 
European Network for the Study of Cholangiocarcinoma (ENSCCA) and participate in the initiative COST Action EURO-CHOLANGIO-NET granted by the COST Association (CA18122).

\section{Footnote}

Conflicts of Interest: The authors have no conflicts of interest to declare.

Ethical Statement: The study was approved by the institutional review board of Inserm (IRB00003888). 


\section{Figure legends}

Figure 1. Identification of a prognostic IncRNA signature in iCCA.

Gene expression profiling of IncRNA was performed from 39 resected iCCA tumors. (A) Venn diagram analysis of IncRNA significantly $(\mathrm{P}<0.05)$ associated with overall $(\mathrm{OS})$ and/or diseasefree (DFS) survival. (B) Example of Kaplan-Meier plots for CDKN2B-AS1 and COL18A1-AS2 expression significantly associated with OS and DFS. (C) Clustering analysis of 39 iCCA samples based on the expression of the 9-IncRNA signature significantly associated with both OS and DFS. Two clusters, A and B, were identified. (D) Kaplan-Meier plots for iCCA samples from cluster $\mathrm{A}$ and cluster $\mathrm{B}$.

Figure 2. CDKN2B-AS1 expression predicts a poor prognosis in several cancers.

(A) Q-RT-PCR of CDKN2B-AS1 was performed in iCCA cases that were further ranked into low $(n=15)$ and high $(n=15)$ expressing tumors. Kaplan-Meier plots for iCCA classified into low vs. high CDKN2B-AS1 expressing tumors. (B) Kaplan-Meier plot analysis (OS) based on the expression of CDKN2B-AS1 in independent gene expression datasets from the TCGA consortium.

Supplementary Figure 1. Histological features of iCCA.

All iCCA tumors $(n=39)$ were analyzed at the histological level on tissue sections $(10 \mu \mathrm{m})$ before RNA extraction and gene expression profiling. Shown are 3 examples of iCCA with low (upper panels), moderate (middle panels) and high (lower panels) stroma abundance, as evidenced by hematoxylin and eosin staining (left panels) and Sirius red staining (right panels). Scale bar: $\underline{250 \mu m}$. 


\section{References}

1. Banales JM, Cardinale V, Carpino G, et al. Expert consensus document: Cholangiocarcinoma: current knowledge and future perspectives consensus statement from the European Network for the Study of Cholangiocarcinoma (ENS-CCA). Nat Rev Gastroenterol Hepatol 2016;13:261-80.

2. Rizvi S, Khan SA, Hallemeier CL, Kelley RK, Gores GJ. Cholangiocarcinoma - evolving concepts and therapeutic strategies. Nat Rev Clin Oncol 2018;15:95-111.

3. Primrose JNF, R.; Palmer, D.H.; Prasad, R.; Mirza, D.; Anthoney, D.A. Adjuvant capecitabine for biliary tract cancer: The BILCAP randomized study. J Clin Oncol 2017;35:4006.

4. Sulpice L, Rayar M, Desille M, et al. Molecular profiling of stroma identifies osteopontin as an independent predictor of poor prognosis in intrahepatic cholangiocarcinoma. Hepatology 2013;58:19922000.

5. Bergeat D, Fautrel A, Turlin B, et al. Impact of stroma LOXL2 overexpression on the prognosis of intrahepatic cholangiocarcinoma. J Surg Res 2016;203:441-50.

6. Merdrignac A, Angenard G, Allain C, et al. A novel transforming growth factor beta-induced long noncoding RNA promotes an inflammatory microenvironment in human intrahepatic cholangiocarcinoma. Hepatol Commun 2018;2:254-69.

7. Wangyang Z, Daolin J, Yi X, et al. NcRNAs and Cholangiocarcinoma. J Cancer 2018;9:100-7.

8. Song W, Miao DL, Chen L. Comprehensive analysis of long noncoding RNA-associated competing endogenous RNA network in cholangiocarcinoma. Biochem Biophys Res Commun 2018;506:1004-12.

9. Wang $\mathrm{X}, \mathrm{Hu} \mathrm{KB}$, Zhang YQ, Yang CJ, Yao HH. Comprehensive analysis of aberrantly expressed profiles of IncRNAs, miRNAs and mRNAs with associated ceRNA network in Cholangiocarcinoma. Cancer Biomark 2018.

10. Han BW, Ye H, Wei PP, et al. Global identification and characterization of IncRNAs that control inflammation in malignant cholangiocytes. BMC Genomics 2018;19:735.

11. Yang W, Li Y, Song X, Xu J, Xie J. Genome-wide analysis of long noncoding RNA and mRNA coexpression profile in intrahepatic cholangiocarcinoma tissue by RNA sequencing. Oncotarget 2017;8:26591-9.

12. Yu Y, Zhang M, Liu J, et al. Long Non-coding RNA PVT1 Promotes Cell Proliferation and Migration by Silencing ANGPTL4 Expression in Cholangiocarcinoma. Mol Ther Nucleic Acids 2018;13:503-13.

13. $\mathrm{Yu} \mathrm{Y}$, Zhang $\mathrm{M}$, Wang $\mathrm{N}$, et al. Epigenetic silencing of tumor suppressor gene CDKN1A by oncogenic long non-coding RNA SNHG1 in cholangiocarcinoma. Cell Death Dis 2018;9:746.

14. Zhang C, Li JY, Tian FZ, et al. Long Noncoding RNA NEAT1 Promotes Growth and Metastasis of Cholangiocarcinoma Cells. Oncol Res 2018;26:879-88.

15. Parasramka M, Yan IK, Wang X, et al. BAP1 dependent expression of long non-coding RNA NEAT1 contributes to sensitivity to gemcitabine in cholangiocarcinoma. Mol Cancer 2017;16:22.

16. Li Y, Cai Q, Li W, Feng F, Yang L. Long non-coding RNA EPIC1 promotes cholangiocarcinoma cell growth. Biochem Biophys Res Commun 2018;504:654-9.

17. Zhang D, Li H, Xie J, et al. Long noncoding RNA LINC01296 promotes tumor growth and progression by sponging miR-5095 in human cholangiocarcinoma. Int J Oncol 2018;52:1777-86.

18. $\mathrm{Xu} \mathrm{Y,} \mathrm{Yao} \mathrm{Y}$, Leng $\mathrm{K}$, et al. Long non-coding RNA UCA1 indicates an unfavorable prognosis and promotes tumorigenesis via regulating AKT/GSK-3beta signaling pathway in cholangiocarcinoma. Oncotarget 2017;8:96203-14.

19. Wang C, Mao ZP, Wang L, et al. Long non-coding RNA MALAT1 promotes cholangiocarcinoma cell proliferation and invasion by activating PI3K/Akt pathway. Neoplasma 2017;64:725-31.

20. Bai JG, Tang RF, Shang JF, Qi S, Yu GD, Sun C. Upregulation of long noncoding RNA CCAT2 indicates a poor prognosis and promotes proliferation and metastasis in intrahepatic cholangiocarcinoma. Mol Med Rep 2018;17:5328-35. 
21. Xia XL, Xue D, Xiang TH, et al. Overexpression of long non-coding RNA CRNDE facilitates epithelial-mesenchymal transition and correlates with poor prognosis in intrahepatic cholangiocarcinoma. Oncol Lett 2018;15:4105-12.

22. $\mathrm{Xu} \mathrm{Y}$, Leng $\mathrm{K}, \mathrm{Li} \mathrm{Z}$, et al. The prognostic potential and carcinogenesis of long non-coding RNA TUG1 in human cholangiocarcinoma. Oncotarget 2017;8:65823-35.

23. Zheng $\mathrm{B}$, Jeong $\mathrm{S}$, Zhu $\mathrm{Y}$, Chen $\mathrm{L}$, Xia $\mathrm{Q}$. miRNA and IncRNA as biomarkers in cholangiocarcinoma(CCA). Oncotarget 2017;8:100819-30.

24. Shi J, Li X, Zhang F, et al. The Plasma LncRNA Acting as Fingerprint in Hilar Cholangiocarcinoma. Cell Physiol Biochem 2018;49:1694-702.

25. Allain C, Angenard G, Clement B, Coulouarn C. Integrative Genomic Analysis Identifies the Core Transcriptional Hallmarks of Human Hepatocellular Carcinoma. Cancer Res 2016;76:6374-81.

26. Coulouarn C, Factor VM, Conner EA, Thorgeirsson SS. Genomic modeling of tumor onset and progression in a mouse model of aggressive human liver cancer. Carcinogenesis 2011;32:1434-40.

27. Lee JS, Chu IS, Heo J, et al. Classification and prediction of survival in hepatocellular carcinoma by gene expression profiling. Hepatology 2004;40:667-76.

28. Sulpice L, Rayar M, Turlin B, et al. Epithelial cell adhesion molecule is a prognosis marker for intrahepatic cholangiocarcinoma. J Surg Res 2014;192:117-23.

29. Chan KM, Tsai $\mathrm{CY}$, Yeh $\mathrm{CN}$, et al. Characterization of intrahepatic cholangiocarcinoma after curative resection: outcome, prognostic factor, and recurrence. BMC Gastroenterol 2018;18:180.

30. Rahnemai-Azar AA, Weisbrod A, Dillhoff M, Schmidt C, Pawlik TM. Intrahepatic cholangiocarcinoma: Molecular markers for diagnosis and prognosis. Surg Oncol 2017;26:125-37.

31. Shen J, Wang W, Wu J, et al. Comparative proteomic profiling of human bile reveals SSP411 as a novel biomarker of cholangiocarcinoma. PLoS One 2012;7:e47476.

32. Sulpice L, Desille M, Turlin B, et al. Gene expression profiling of the tumor microenvironment in human intrahepatic cholangiocarcinoma. Genom Data 2016;7:229-32.

33. Yan Y, Li S, Wang S, et al. Long noncoding RNA HAND2-AS1 inhibits cancer cell proliferation, migration, and invasion in esophagus squamous cell carcinoma by regulating microRNA-21. J Cell Biochem 2018.

34. Zhou J, Lin J, Zhang H, Zhu F, Xie R. LncRNA HAND2-AS1 sponging miR-1275 suppresses colorectal cancer progression by upregulating KLF14. Biochem Biophys Res Commun 2018;503:1848-53.

35. Gao C, Lu W, Lou W, Wang L, Xu Q. Long noncoding RNA HOXC13-AS positively affects cell proliferation and invasion in nasopharyngeal carcinoma via modulating miR-383-3p/HMGA2 axis. J Cell Physiol 2018.

36. Pasmant E, Laurendeau I, Heron D, Vidaud M, Vidaud D, Bieche I. Characterization of a germ-line deletion, including the entire INK4/ARF locus, in a melanoma-neural system tumor family: identification of ANRIL, an antisense noncoding RNA whose expression coclusters with ARF. Cancer Res 2007;67:39639.

37. Kong Y, Hsieh CH, Alonso LC. ANRIL: A IncRNA at the CDKN2A/B Locus With Roles in Cancer and Metabolic Disease. Front Endocrinol (Lausanne) 2018;9:405.

38. Wang $\mathrm{H}$, Liu Y, Zhong J, et al. Long noncoding RNA ANRIL as a novel biomarker of lymph node metastasis and prognosis in human cancer: a meta-analysis. Oncotarget 2018;9:14608-18.

39. Hua L, Wang CY, Yao KH, Chen JT, Zhang JJ, Ma WL. High expression of long non-coding RNA ANRIL is associated with poor prognosis in hepatocellular carcinoma. Int J Clin Exp Pathol 2015;8:307682.

40. Aguilo F, Zhou MM, Walsh MJ. Long noncoding RNA, polycomb, and the ghosts haunting INK4bARF-INK4a expression. Cancer Res 2011;71:5365-9. 
41. Nie FQ, Sun M, Yang JS, et al. Long noncoding RNA ANRIL promotes non-small cell lung cancer cell proliferation and inhibits apoptosis by silencing KLF2 and P21 expression. Mol Cancer Ther 2015;14:268-77.

42. Zhang EB, Kong R, Yin DD, et al. Long noncoding RNA ANRIL indicates a poor prognosis of gastric cancer and promotes tumor growth by epigenetically silencing of miR-99a/miR-449a. Oncotarget 2014;5:2276-92.

43. Qiu JJ, Wang Y, Liu YL, Zhang Y, Ding JX, Hua KQ. The long non-coding RNA ANRIL promotes proliferation and cell cycle progression and inhibits apoptosis and senescence in epithelial ovarian cancer. Oncotarget 2016;7:32478-92.

44. Zhu H, Li X, Song Y, Zhang P, Xiao Y, Xing Y. Long non-coding RNA ANRIL is up-regulated in bladder cancer and regulates bladder cancer cell proliferation and apoptosis through the intrinsic pathway. Biochem Biophys Res Commun 2015;467:223-8.

45. Zou ZW, Ma C, Medoro L, et al. LncRNA ANRIL is up-regulated in nasopharyngeal carcinoma and promotes the cancer progression via increasing proliferation, reprograming cell glucose metabolism and inducing side-population stem-like cancer cells. Oncotarget 2016;7:61741-54.

46. Zhao JJ, Hao S, Wang LL, et al. Long non-coding RNA ANRIL promotes the invasion and metastasis of thyroid cancer cells through TGF-beta/Smad signaling pathway. Oncotarget 2016;7:57903-18.

47. Ma J, Li T, Han X, Yuan H. Knockdown of LncRNA ANRIL suppresses cell proliferation, metastasis, and invasion via regulating miR-122-5p expression in hepatocellular carcinoma. J Cancer Res Clin Oncol 2018;144:205-14.

48. Xu R, Mao Y, Chen K, He W, Shi W, Han Y. The long noncoding RNA ANRIL acts as an oncogene and contributes to paclitaxel resistance of lung adenocarcinoma A549 cells. Oncotarget 2017;8:3917784.

49. Lin L, Gu ZT, Chen WH, Cao KJ. Increased expression of the long non-coding RNA ANRIL promotes lung cancer cell metastasis and correlates with poor prognosis. Diagn Pathol 2015;10:14.

50. Xie $Y$, Zhang $Y$, Du $L$, et al. Circulating long noncoding RNA act as potential novel biomarkers for diagnosis and prognosis of non-small cell lung cancer. Mol Oncol 2018;12:648-58.

51. Soares do Amaral N, Cruz EMN, de Melo Maia B, Malagoli Rocha R. Noncoding RNA Profiles in Tobacco- and Alcohol-Associated Diseases. Genes (Basel) 2016;8.

52. Wan G, Mathur R, Hu X, et al. Long non-coding RNA ANRIL (CDKN2B-AS) is induced by the ATME2F1 signaling pathway. Cell Signal 2013;25:1086-95.

53. Lu Y, Zhou X, Xu L, Rong C, Shen C, Bian W. Long noncoding RNA ANRIL could be transactivated by c-Myc and promote tumor progression of non-small-cell lung cancer. Onco Targets Ther 2016;9:307784.

54. Rodriguez C, Borgel J, Court F, Cathala G, Forne T, Piette J. CTCF is a DNA methylation-sensitive positive regulator of the INK/ARF locus. Biochem Biophys Res Commun 2010;392:129-34. 


\section{Figure [1]}

Click here to download high resolution image

A

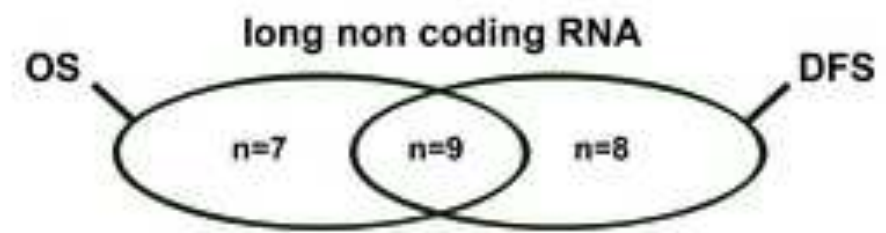

B

OS (CDKN2B-AS1)

DFS (CDKN2B-AS1)
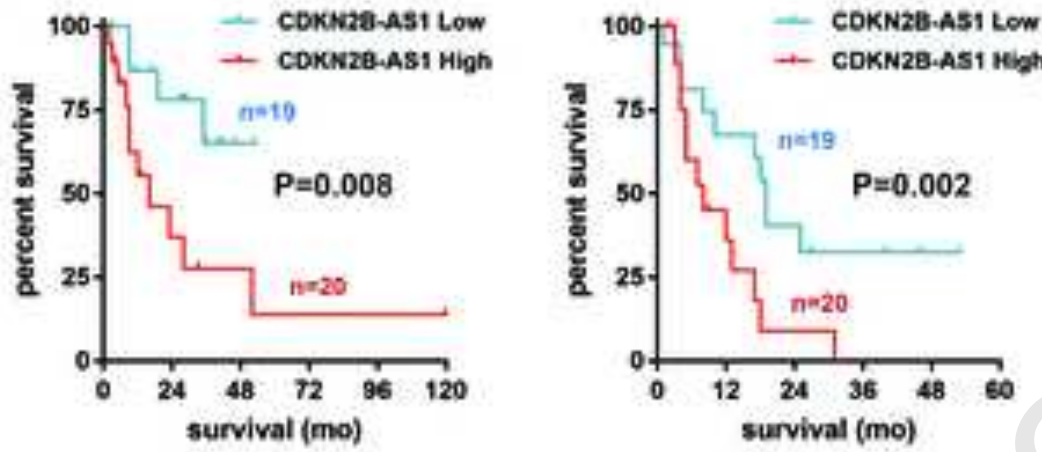

OS (COL18A1-AS2)

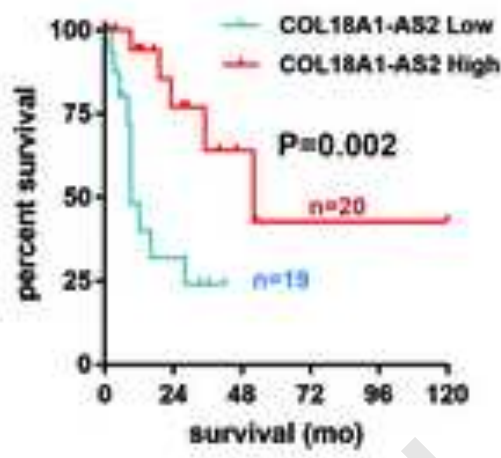

DFS (COL18A1-AS2)

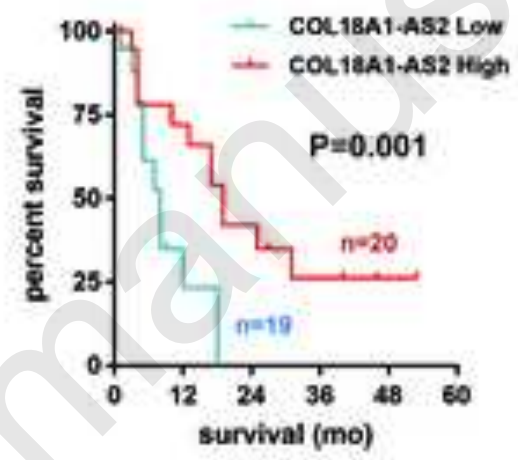

C

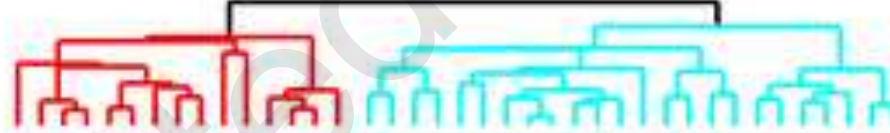
CUISIERA
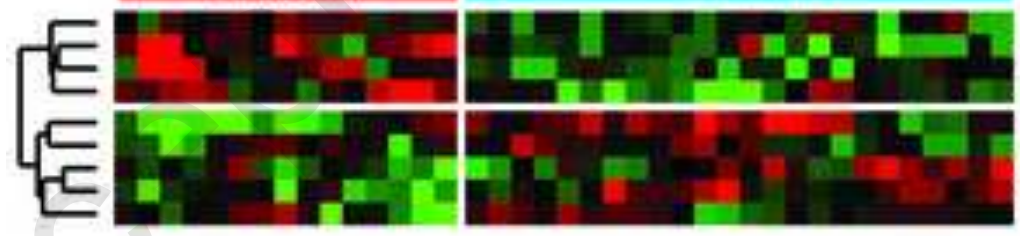

incecoks-1. COKNZE-AST moxct3ats noC_o 0004 S InE-OCHCREH incereuiz.s inceniss CoLtsAt-Ast Ine-24t 121215.241

OS (ncRNA signature)

DFS (ncRNA signature)

D
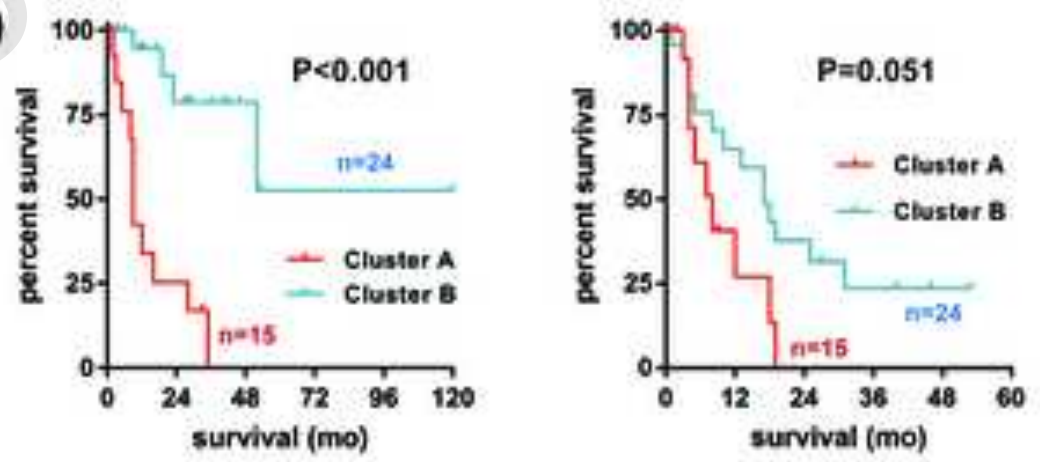
A

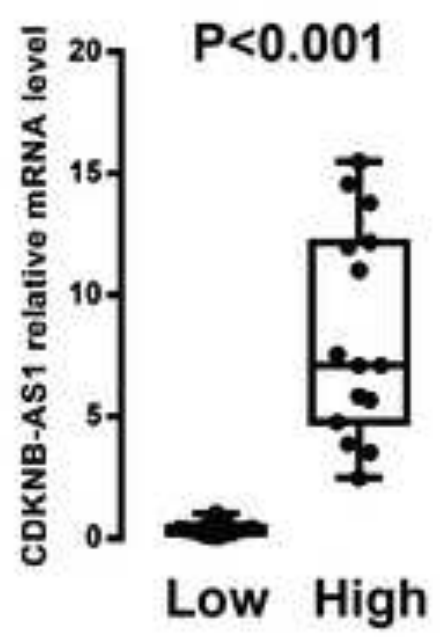

OS (CDKN2B-AS1)

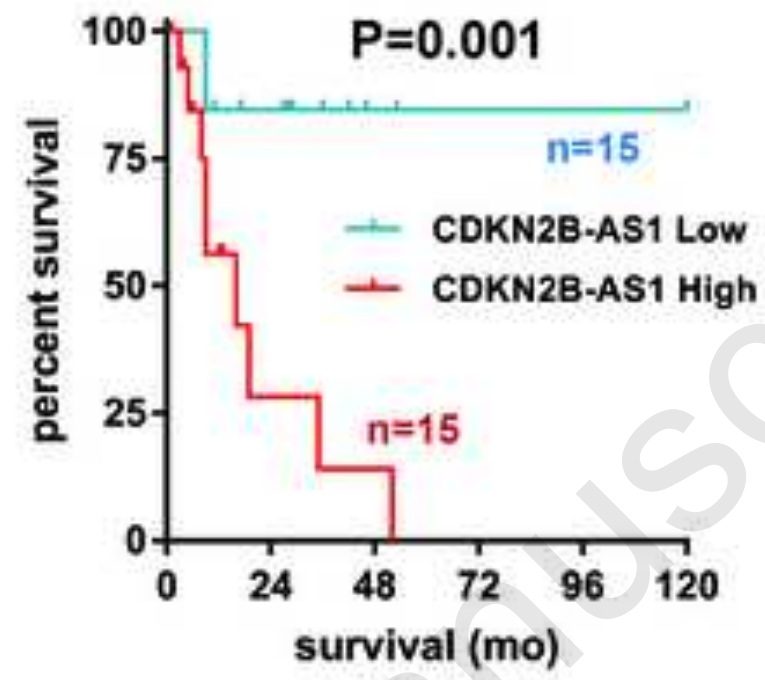

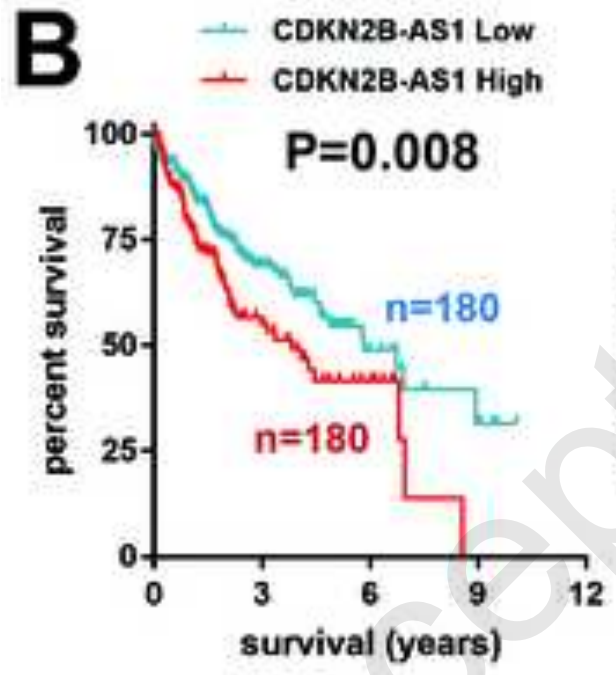

LIHC: Liver

hepatocellular carcinoma
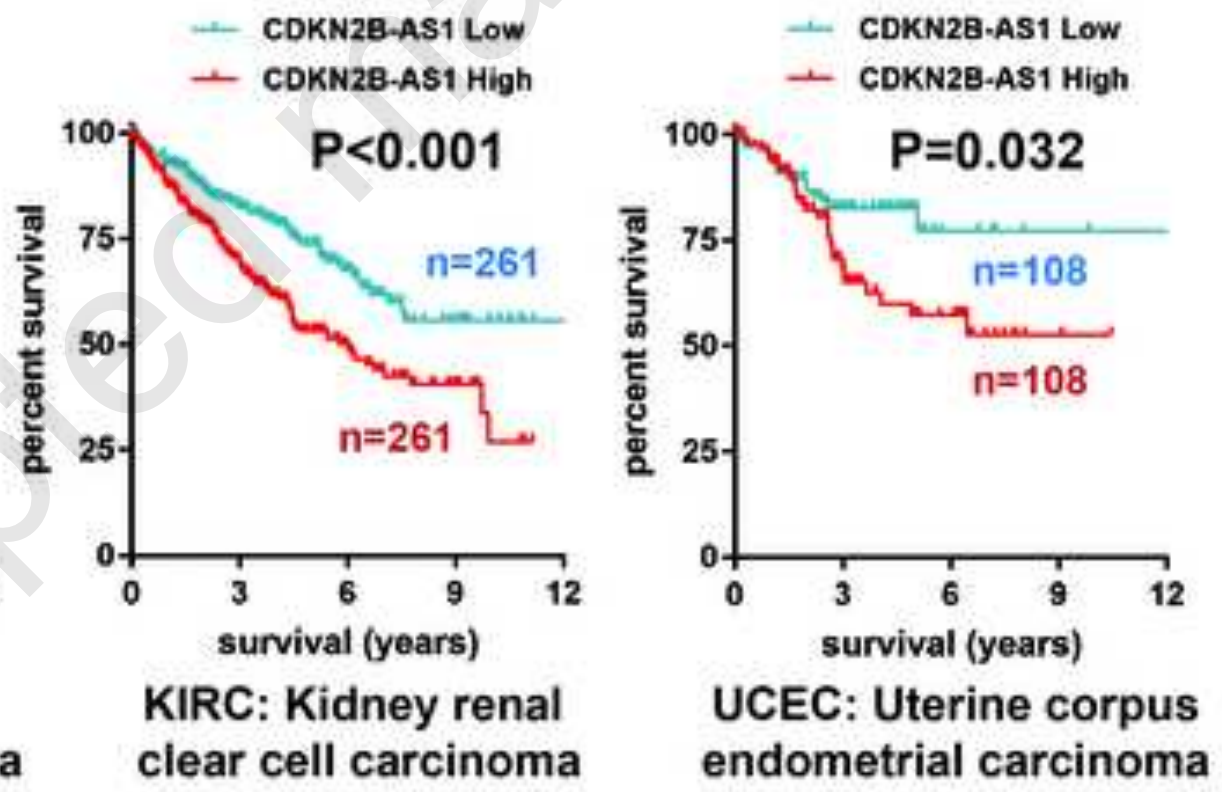
Table 1. Clinical and pathological features of iCCA patients.

Clinical and pathological features $\quad n=39$

Age (years, mean $\pm S D)$

$61 \pm 14$

Gender (male/female)

$27 / 12$

$\mathrm{BMI}$

$26 \pm 3$

Tobacco use

$18(46 \%)$

Diabetes

$7(18 \%)$

Etiology

HBV or HCV infection

$5(13 \%)$

Alcohol

$3(8 \%)$

Hemochromatosis

$1(3 \%)$

Primary biliary cirrhosis

$1(3 \%)$

Histologically normal liver

$3(8 \%)$

Association of factors

$6(15 \%)$

Undetermined

$20(51 \%)$

Metavir fibrosis score (F0/F1/F2/F3/F4/na)

$7 / 6 / 6 / 4 / 6 / 10$

Cirrhosis

$6(15 \%)$

Steatosis

$21(54 \%)$

Tumor size ( $\mathrm{mm}$, mean $\pm \mathrm{SD})$

$63 \pm 27$

Tumor $\geq 50 \mathrm{~mm}$

$27(69 \%)$

OMS differentiation grade

Well-differentiated

$15(38 \%)$

Moderately-differentiated

$17(44 \%)$

Poorly-differentiated

$7(18 \%)$ 


$\begin{array}{lc}\text { Satellite nodules }>1 & 19(49 \%) \\ \text { Tumor necrosis } & 16(41 \%) \\ \text { Microvascular tumor invasion } & 20(51 \%) \\ \text { Portal invasion } & 6(15 \%) \\ \text { Biliary tract invasion } & 10(26 \%) \\ \text { Perineural invasion } & 15(38 \%) \\ \text { Capsular invasion } & 8(21 \%) \\ \text { Lymph nodes invasion } & 11(28 \%) \\ \text { Extrahepatic tissue invasion } & 10(26 \%)\end{array}$


Table 2. IncRNA signature predictive of overall (OS) and disease-free (DFS) survival.

OS

DFS

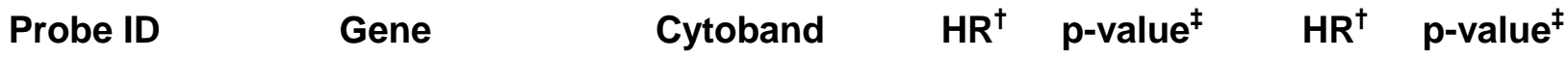

IncRNA up-regulated in poor prognosis iCCA

$\begin{array}{lllllll}\text { A_21_P0006229 Inc-CDK9-1 } & \text { hs } \mid 9 q 34.11 & 3.83 & 0.007 & 3.65 & 0.030\end{array}$

A_21_P0012328 XLOC_I2_009441 hs|22q11.1 $1.99 \quad 0.018 \quad 2.01 \quad 0.010$

$\begin{array}{lllllll}\text { A_19_P00322815 } & \text { CDKN2B-AS1 } & \text { hs } \mid 9 p 21.3 & 1.86 & 0.008 & 2.06 & 0.002\end{array}$

$\begin{array}{lllllll}\text { A_22_P00019478 HOXC13-AS } & \text { hs|12q13.13 } & 1.83 & 0.047 & 1.75 & 0.048\end{array}$

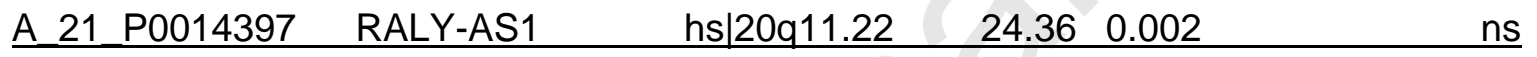

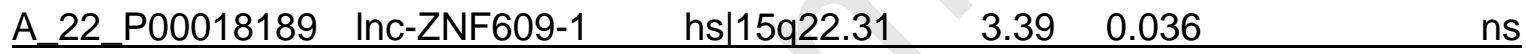

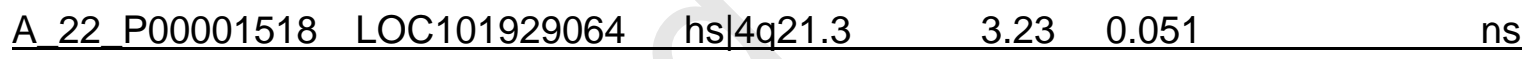

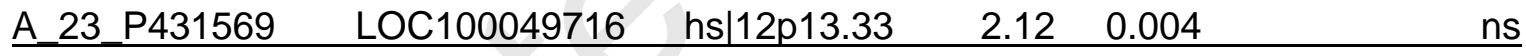

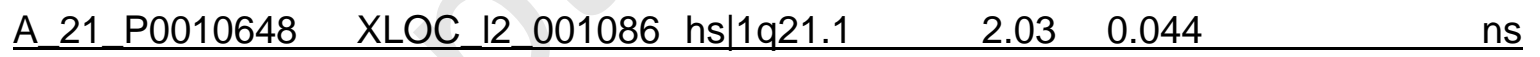

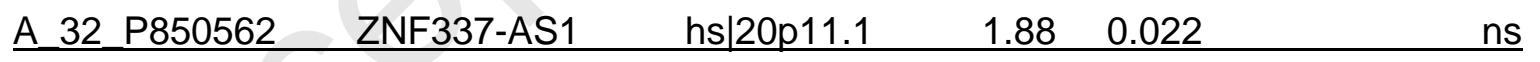

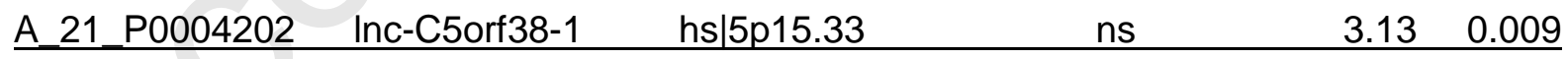

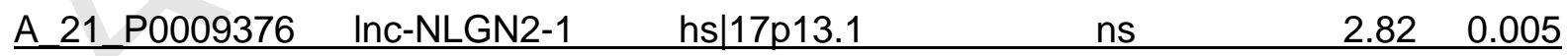

IncRNA down-regulated in poor prognosis iCCA

$\begin{array}{lllllll}\text { A_21_P0005160 Inc-CCHCR1-1 hs|6p21.33 } & 0.72 & 0.001 & 0.71 & 0.005\end{array}$

$\begin{array}{lllllll}\text { A_24_P312325 Inc-AF131215.3.1 hs|8p23.1 } & 0.65 & 0.005 & 0.56 & 0.000\end{array}$ 

A_21_P0003146 Inc-CBLB-5
hs|3q13.12
$0.60 \quad 0.020$
$0.56 \quad 0.024$

$\begin{array}{lllllll}\text { A_22_P00025222 } & \text { COL18A1-AS2 } & \text { hs|21q22.3 } & 0.55 & 0.002 & 0.55 & 0.001\end{array}$

$\begin{array}{lllllll}\text { A_22_P00012968 } & \text { Inc-RELL2-1 } & \text { hs } \mid 5 q 31.3 & 0.39 & 0.003 & 0.38 & 0.001\end{array}$

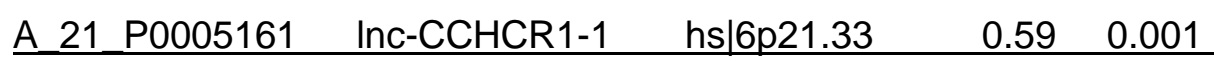

ns

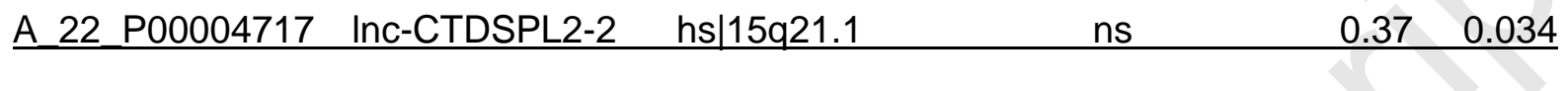

\begin{tabular}{llllll} 
A 22 & P00014082 HAND2-AS1 & hs $\mid 4 q 34.1$ & ns & 0.46 & 0.049 \\
\hline
\end{tabular}

$\begin{array}{lllllll}\text { A } 22 & \text { P00015084 } & \text { Inc-SNURF-3 } & \text { hs } \mid 15 q 11.2 & \text { ns } & 0.49 & 0.014\end{array}$

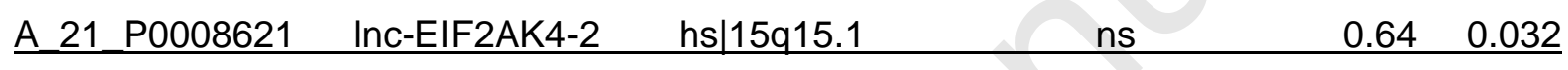

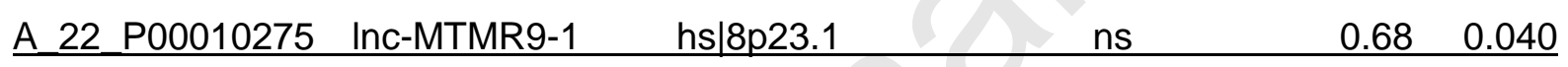

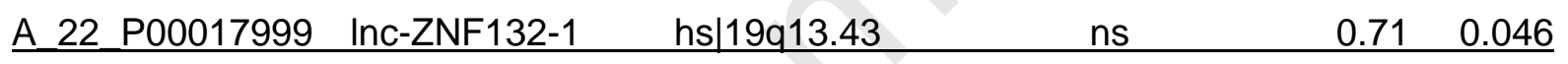

Footnotes: ${ }^{\dagger}$ Hazard Ratio; ${ }^{\ddagger}$ p-value, as determined by a log-rank test; ns: not significant 
Footnotes: ${ }^{\dagger}$ number of patients in each tested group $(n=15$ patients and $n=24$ patients for clusters A and B, respectively; although clinical data were missing for some patients); ${ }^{\ddagger} p$-value, as determined by a Fisher's exact test 
Footnotes: ${ }^{\dagger}$ number of patients in each tested group $(n=15$ patients per group although clinical data were missing for some patients); ${ }^{\ddagger}$ p-value, as determined by a Fisher's exact test 